\title{
Genome-wide identification of the context- dependent sRNA expression in Mycobacterium tuberculosis
}

\author{
Vimla Kany G. Ami ${ }^{\dagger}$, Rami Balasubramanian ${ }^{\dagger}$ and Shubhada R. Hegde ${ }^{*}$ (D)
}

\begin{abstract}
Background: Tuberculosis remains one of the leading causes of morbidity and mortality worldwide. Therefore, understanding the pathophysiology of Mycobacterium tuberculosis is imperative for developing new drugs. Posttranscriptional regulation plays a significant role in microbial adaptation to different growth conditions. While the proteins associated with gene expression regulation have been extensively studied in the pathogenic strain $M$. tuberculosis H37Rv, post-transcriptional regulation involving small RNAs (sRNAs) remains poorly understood.

Results: We developed a novel moving-window based approach to detect sRNA expression using RNA-Seq data. Overlaying ChIP-seq data of RNAP (RNA Polymerase) and NusA suggest that these putative sRNA coding regions are significantly bound by the transcription machinery. Besides capturing many experimentally validated sRNAs, we observe the context-dependent expression of novel sRNAs in the intergenic regions of $M$. tuberculosis genome. For example, ncRv11806 shows expression only in the stationary phase, suggesting its role in mycobacterial latency which is a key attribute to long term pathogenicity. Also, ncRv11875C showed expression in the iron-limited condition, which is prevalent inside the macrophages of the host cells.
\end{abstract}

Conclusion: The systems level analysis of sRNA highlights the condition-specific expression of sRNAs which might enable the pathogen survival by rewiring regulatory circuits.

Keywords: sRNA, RNA-Seq, Tuberculosis, Gene regulation, Persistence

\section{Background}

Tuberculosis remains one of the most successful human pathogens and one among the top 10 leading causes of morbidity and mortality from infectious diseases worldwide [1]. In about $90 \%$ of the affected individuals, bacteria may persist in the form of an asymptomatic latent infection, which may reactivate under any form of immunosuppression [2]. During the course of infection, M. tuberculosis adapts to different micro-environments such as iron restriction, starvation, hypoxia and low $\mathrm{pH}$. The transcriptional and translational machinery associated with bacterial adaptation in response to environmental changes have been widely studied in M. tuberculosis [3]. Most of these studies have invariably focused on the

\footnotetext{
* Correspondence: shubhada@ibab.ac.in

${ }^{\dagger}$ Vimla Kany G. Ami and Rami Balasubramanian contributed equally to this work.

Institute of Bioinformatics and Applied Biotechnology (IBAB), Bengaluru 560 100, India
}

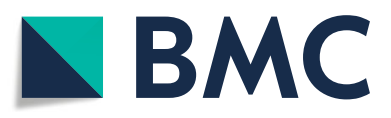

(๑) The Author(s). 2020 Open Access This article is distributed under the terms of the Creative Commons Attribution 4.0 International License (http://creativecommons.org/licenses/by/4.0/), which permits unrestricted use, distribution, and

reproduction in any medium, provided you give appropriate credit to the original author(s) and the source, provide a link to the Creative Commons license, and indicate if changes were made. The Creative Commons Public Domain Dedication waiver (http://creativecommons.org/publicdomain/zero/1.0/) applies to the data made available in this article, unless otherwise stated. protein-coding regions of the genome. However, with the emergence of transcriptome sequencing revolution, expression patterns in the non-protein coding regions can be analysed meaningfully. This is important as the recent studies suggest that bacterial genomes code for small non-coding RNAs (sRNAs), which play a significant role in modulating translation or mRNA stability [4].

Depending on the base pairing with their target mRNAs, there are three broad classes of sRNAs (i) Antisense sRNAs which are present in the opposite strand of their target mRNA and share an extensive sequence complementarity, (ii) Trans-encoded sRNAs which are located largely in the intergenic regions (IGRs) and share limited sequence complementarity with their target mRNAs, and (iii) Cis-encoded sRNAs which are present in the untranslated regions (UTRs) of the genes [4-6]. These sRNAs are important for adapting to various stresses and environmental changes caused by the host 
defense mechanisms. For example, Escherichia coli sRNA RyhB maintains iron homeostasis by downregulating the expression of iron utilization proteins such as SodB, PflA and MsrB [7]. Also in E. coli, sRNAs DsrA and RprA base-pair with the mRNA of the stress response sigma factor rpoS, thereby increasing its stability by rendering protection from RNaseE [8]. While adapting to less carbon source, sRNA CrcZ in Pseudomonas aeruginosa helps in relieving catabolite repression by sequestering Crc protein [9]. Some of the sRNAs identified in M. tuberculosis so far include F6, B11, MTS2823, ncRV12659, DrrS, Mcr7 and MrsI [10-15]. DrrS showed increased expression in the stationary phase which is regulated by the $M$. tuberculosis dormancy regulator DosR [12]. Also, ncRv12659 which is transcribed anti sense to the gene $R v 2660 c$, was shown to be expressed during starvation [15]. Another condition-specific sRNA is MrsI which is expressed during iron starvation, oxidative stress and membrane stress [14].

Both sequence and expression based approaches have been used to identify sRNAs in the bacterial genomes. Previously in E. coli, putative sRNAs were shortlisted by identifying conserved IGRs across Salmonella typhi, S. paratyphi, and S. Typhimurium, with potential transcription start and termination sites [16]. In another sequence-based approach, 13 well annotated bacterial species were considered for identifying conserved IGRs which are likely to code for sRNAs [17]. However, such sequence-based methods remain efficient in identifying only those sRNAs which are conserved across other bacterial genomes. The emergence of transcriptome data has opened a way to identify sRNAs based on their expression. In E. coli, IGRs were identified as sRNAs if they showed significant expression compared to the upstream and downstream protein-coding genes [18]. In $M$. tuberculosis, expression data corresponding to the log-phase growth was utilised to identify sRNAs by considering the read depth at a given position in the genome excluding the UTRs [19]. However, none of these genome-wide analyses focused on the conditional expression of the sRNAs in different stress environments.

While sequence conservation-based approaches fail to identify species specific sRNAs and sRNAs which show significant divergence, expression based methods need to be improved to address the following challenges: a) to detect sRNA expression independent of the neighbouring gene expression and the signals arising due to UTR expression, b) to identify sRNA expression within an IGR without normalising the reads across the entire IGR, and c) to identify condition-specific expression of the sRNAs.

We used RNA-Seq data to identify sRNAs in $M$. tuberculosis. Our method employs sliding-windows along the IGRs to detect sRNA expression, while efficiently dismissing the expression signals arising from the upstream and the downstream genes and their UTRs. Previously, such moving windows of normalised RNA-Seq values along the genome were used to detect sigma-H dependent promoters in Listeria monocytogenes [20]. Also, as sRNAs are recognised to tune the cellular responses, we profiled the conditional expression of sRNAs by analysing the expression data across multiple growth conditions. Analysis of these condition specific sRNAs along with their predicted targets provided insights on the putative regulatory mechanisms for bacterial adaptation under various stress conditions.

\section{Results and discussion}

\section{Profiling expression data of $M$. tuberculosis to identify sRNAs}

We used RNA-Seq data of the mid-exponential phase culture to develop a methodology for identifying sRNAs in the intergenic regions (IGRs) of M. tuberculosis [21]. Initially, we quantified the expression of different functional elements in the genome and tested if the RNASeq data is sufficient to detect the IGR sRNA expression. Of the 4018 protein coding genes (CDS), 1000 highly expressing (HE) and less expressing (LE) genes respectively, were extracted (Additional file 9: Tables S3a and S3b). These were compared with the essential genes which show high expression levels [22-25] (Additional file 1: Figure S1). rRNAs are the abundantly expressed RNA species in the cell. Similarly, tRNAs show significant expression, which is comparable to the expression of the essential genes (Additional file 1: Figure S1). Further, we divided the IGR into untranslated regions (UTRs) and the absolute intergenic region (AbIGR) which is devoid of the UTRs. Both IGRs and AbIGRs show significant expression which is higher than the less expressed genes, implying functional importance of the non-protein coding regions in the genome. The high expression levels of the experimentally validated sRNAs encoded in the IGRs suggest that RNA-Seq could be potentially used to predict the location as well as the expression levels of sRNAs in the bacterial genomes (Additional file 8: Table S2; Additional file 1: Figure S1).

About 1037 IGRs of length more than $100 \mathrm{bp}$ which are devoid of repeat regions, insertion elements, rRNAs and tRNAs were considered for identifying sRNAs (Methods). The distribution of these IGRs shows varied lengths ranging from 100 to 1500 bases (Additional file 2: Figure S2). As the given sRNA is unlikely to span the entire length of the IGR, a moving window approach was adopted to capture the expression of the sRNAs. The IGRs were covered by the windows of lengths 50 bases with 25 bases sliding and the expression of each of these windows was compared to its neighbouring windows to identify the peak expression signal. A window with an expression value of more than three times the 
median expression value of all the IGRs and showing higher expression compared to its adjacent windows was considered as a potential sRNA encoding region (Additional file 3: Figure S3; Methods).

Using this approach, we identified 119 IGR regions as significantly expressed in the mid-exponential growth phase which are likely to encode sRNA (Additional file 10: Table S4a). Of these, 52 and 2 expression regions were from the $5^{\prime}$ and the $3^{\prime} U T R s$ of the neighbouring genes, respectively. These included three experimentally validated sRNAs, namely, ncRv13003Ac, ncRv3418Ac and ncRv13660Ac which are in the 5'UTR of their respective neighbouring genes. The rest 65 expression regions were localised in the AbIGR which included experimentally validated sRNAs ncRv11147Ac, ncRV2395, ncRv11534A and ncRv11846c [10, 11, 26].

\section{Increased transcription machinery binding in the expressed IGRs}

For the 119 potential sRNA regions identified in the mid-exponential growth phase, we tested their expression by profiling the binding of RNA polymerase (RNAP) and NusA along these regions. RNA polymerase (RNAP) is the principal enzyme involved in synthesising of RNA from a DNA template. Another member of the transcription complex is NusA, a terminator and an anti-terminator of transcription which was shown to facilitate transcription by binding to RNAP in both midexponential and stationary phases of growth [27-29].

ChIP-seq data of RNAP and NusA in M. tuberculosis were used to test if these transcription-associated proteins are significantly bound to the identified sRNA regions on the genome [27]. Additionally, ChIP-seq data of the polyketide synthase regulator Rv1186c and a genomic control sample attributing non-specific binding signals across the genome were used as control datasets (SRR1524124 and SRR5753731). We observe that the 119 expressed sRNA regions were significantly bound by both RNAP and NusA compared to the non-expressed IGRs ( $\mathrm{P}$ value $<7.702 \mathrm{e}-10$ and $\mathrm{P}$ value $<7.208 \mathrm{e}-06$, respectively). Expression analysis using RNA-Seq data associated with RNAP and NusA experiments (SRP015746) showed that these putative sRNA regions are highly expressed compared to the non-expressed IGRs (P value $<4.424 \mathrm{e}-07$ ). However, such a differential binding was not observed in the control samples Rv1186c and the genomic control ( $P$ value $<0.3239$ and $\mathrm{P}$ value $<0.7858$ ). Further, we performed a similar analysis for the highly expressed protein coding genes and the less expressed protein coding genes. As expected, the highly expressed protein coding genes showed increased binding of RNAP and NusA compared to the control samples (P-value <2.2e-16) (Fig. 1). Therefore, sRNA regions identified in the mid-exponential growth phase seem to be significantly bound by the transcription machinery, suggesting transcriptional activity in these genomic regions.

\section{Context-dependent expression of sRNAs to revamp cellular responses}

Similar to transcription factors, bacterial sRNAs show condition-specific expression which enables them to impart necessary cellular responses for a particular growth environment $[7,9,12,15]$. The 119 sRNAs described earlier were identified in the mid-exponential phase growth culture. To gain more insights on the contextdependent expression of the sRNAs, we analysed $M$. $t u$ berculosis RNA-Seq data of 5 different studies representing 15 different growth conditions. These included exponential and stationary growth phases, M. tuberculosis persistence and reactivation conditions, stress conditions such as iron depletion, NO treatment and acidic $\mathrm{pH}$ growth [27, 30, 31] (Additional file 8: Table S1). Of the 1037 IGRs considered in the analysis, we observed the expression of 430 putative sRNAs from 361 IGRs in at least one growth condition (Additional file 10: Table S4b; Additional file 4: Figures. S4a and S4b). Genomewide representation of the IGR sRNA expression highlights the context-dependent expression of sRNAs along the $M$. tuberculosis genome (Figure 2).

We captured the expression of 24 sRNAs from 42 experimentally validated intergenic sRNAs of $M$. tuberculosis (Table 1). Over-expression of the sRNA MTS2823 (ncRv13661) was previously shown to affect the growth rate of M. tuberculosis [11]. In our analysis, MTS2823 showed expression in all the 15 growth conditions with a very high expression in the stationary phase (Fig. 3). One of the promising targets predicted for MTS2823 is the gene Rv0115 (hddA) which codes for D-alpha-Dheptose-7-phosphate kinase, involved in GDP-L-fucose salvage pathway. Also, some of the other targets predicted for MTS2823 such as hemD, Rv0875, ribH, mpt83, Rv3828c and Rv3839 were down-regulated by $\geq 2.5$-fold upon over expression of MTS2823 [11]. Another sRNA MrsI (ncRv11846) was shown to be induced during exposure to iron starvation, oxidative stress and membrane stress. MrsI represses the iron storage mRNA $b f r A$ in iron deprived conditions [14]. Along similar lines, we observe the induced expression of MrsI during iron limiting conditions. Also, MrsI expression is significantly induced in the late stages of iron deprivation compared to low iron day-1 ( $P$ value $<2.2 \mathrm{e}-16$ ) (Fig. 4).

While 6 sRNAs are expressed irrespective of the growth condition, the rest of the sRNAs showed context-dependent expression (Fig. 2 and Additional file 4: Figure. S4b). The sRNA ncRv11806, which is flanked by the genes PE20 and PPE32, showed expression only in the stationary phase of growth $(P$ value $<2.2 \mathrm{e}-16)$ 


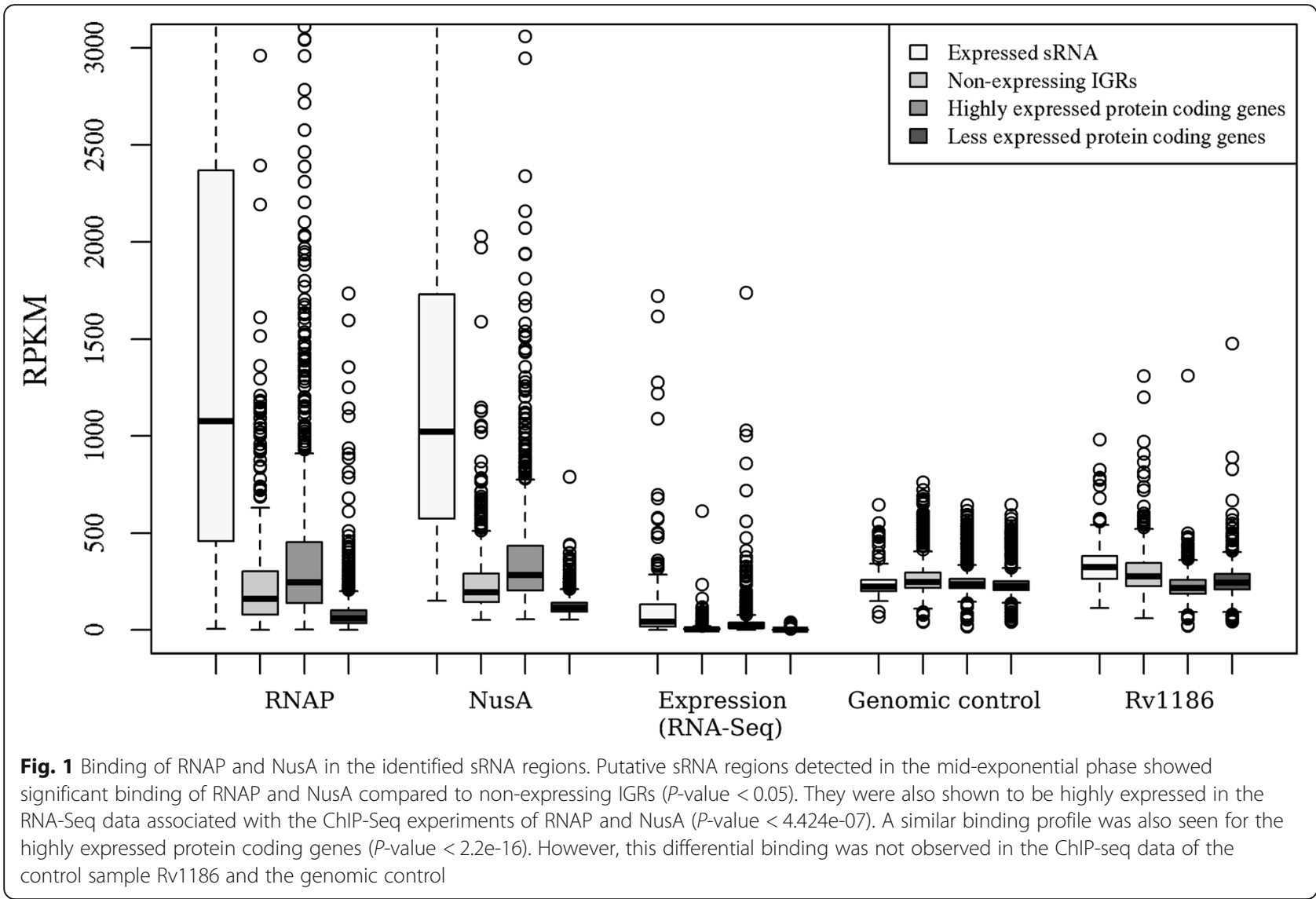

(Fig. 5). Some of the potential targets predicted for this region were $r p f B, n r d H$, memE, thyX, senX 3 and mutT1 (Additional file 11: Table S5a). The expression of $r p f B$ which codes for a resuscitation promoting factor (RpfB) gets diminished as the pathogen transits into stationary phase [32] (Additional file 5: Figure S5A). ncRv11806 is predicted to bind at the 5'UTR region of $r p f B$ mRNA, which extends further to the protein coding region (Additional file S5: Figure S5B). We therefore hypothesise that the induced expression of ncRv11806 in the stationary phase of $M$. tuberculosis growth might repress the translation of $r p f B$.

Another sRNA ncRv11875C located between the genes Rv1875 and bfrA showed significant expression in the iron limiting conditions ( $\mathrm{P}$ value <2.2e-16) (Fig. 6). Genes Rv3003c, Rv1924c, Rv3150, Rv1937, Rv1728c, $R v 1626, R v 1308, R v 0544 c, R v 0532$ and $R v 1526 c$ are the predicted targets for this sRNA (Additional file 11: Table S5b). Subsequent gene expression analysis revealed that the predicted targets Rv1308, Rv3003c, Rv1924c, $R v 1728 c, R v 1626$ and $R v 3150$ showed reduced expression in the iron limited condition compared to midexponential and high iron growth conditions (Additional file 6: Figure S6). On the other hand, Rv1937, which is a probable monooxygenase containing [2Fe-2S] cluster shows increased expression in the iron limited conditions, suggesting a probable positive regulation by ncRv11875C (Additional file 6: Figure S6).

M. tuberculosis persists in the host with a reduced metabolic activity and gets reactivated upon encountering favourable conditions for growth [2]. However, the regulatory roles of mycobacterial sRNAs in these growth phases remain poorly understood. We observe that the sRNA ncRv11706A, which is in the intergenic region between $R v 1706 A$ and $R v 1706 c$, is highly expressed in hypoxia induced persistence $(P$ value $<2.2 \mathrm{e}-16)$ (Fig. 7). Some of the predicted targets shortlisted for this sRNA such as $R v 3158, R v 2736 c, R v 1382, R v 2325 c$ and $R v 2898 c$ showed reduced expression in persistence compared to midexponential growth and various reactivation phases (Additional file 11: Table S5c; Additional file 7: Figure S7A). On the other hand, predicted target genes $R v 3047 c$ and $R v 3102 c$ showed increased expression in persistence compared to mid-exponential and reactivation conditions (Additional file 7: Figure S7A). Among these, Rv2736c encoding RecX was repressed in persistence significantly. RecX, which modulates the activity of RecA by inhibiting its ATP hydrolysis and the strand-exchange activities, was shown to be significantly downregulated in SS18b model which mimics latent TB infection [33, 34]. ncRv11706A is 


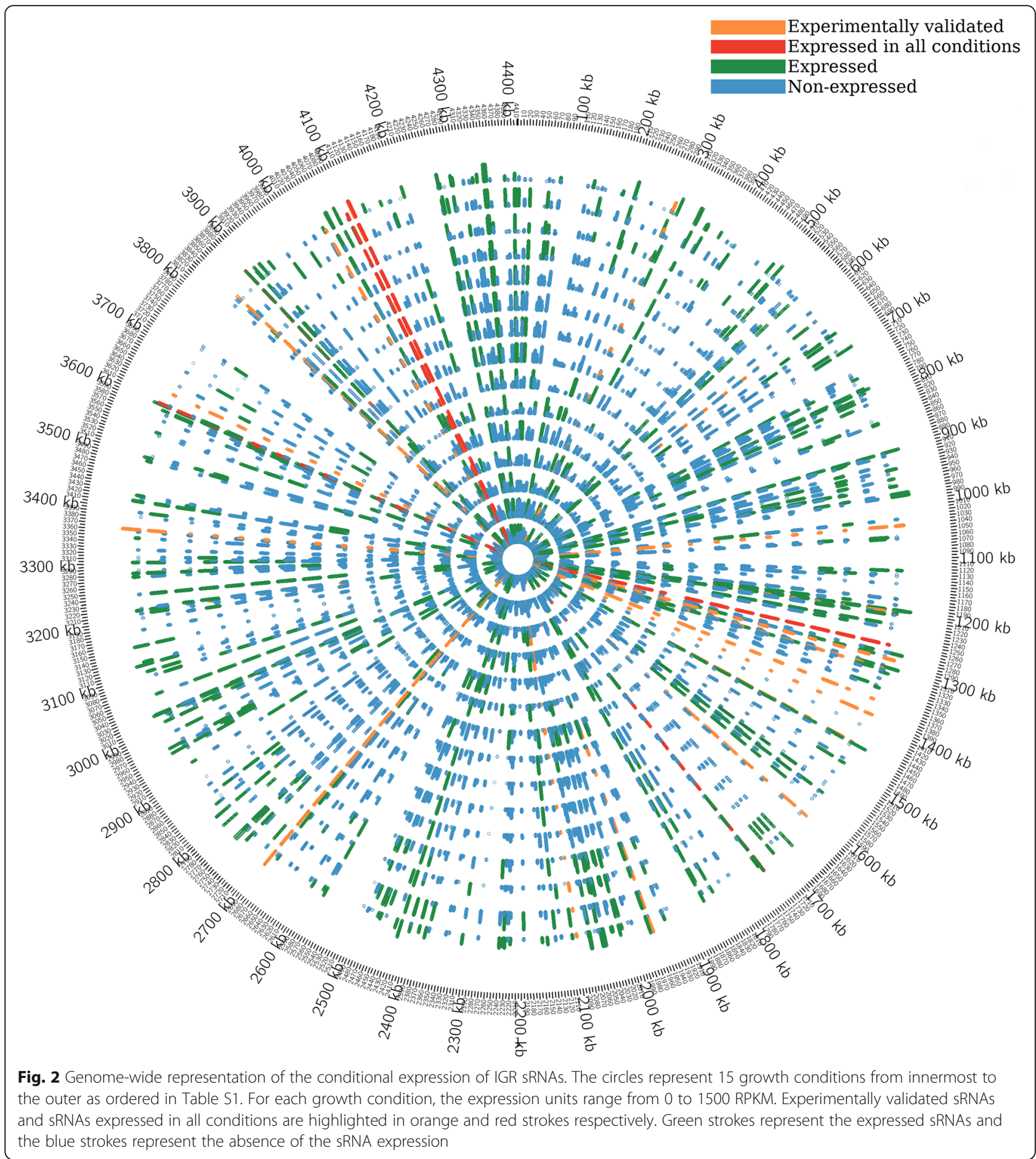

predicted to interact at 3 bases downstream of the start codon of recX mRNA, suggesting that this interaction might affect the translation, thereby repressing $\operatorname{RecX}$ activity (Additional file 7: Figure S7B). These conditionally expressed sRNAs in the context of their predicted targets and the functions, therefore, provide insights on bacterial adaptability to changing growth environments.

\section{Conclusions}

Bacterial genomes encode both cis and trans-acting sRNAs which are important for the regulation of cellular functions [4]. Since sequence conservation is poor for the sRNAs across species, homology-based methods are less powerful in identifying sRNAs [35]. Previously, there were attempts to determine sRNA coding regions in the 
Table 1 Conditional expression of the 24 experimentally validated sRNAs. Of these, only 2 sRNAs were expressed in all the 15 growth conditions

\begin{tabular}{|c|c|c|c|c|c|}
\hline sRNA & Start & End & Strand & Number of Expressed Conditions & PubMed Identifier \\
\hline ncRv10243A (F6) & 293604 & 293705 & + & 12 & 23284830 \\
\hline ncRv10537A & 629877 & 629975 & + & 6 & $19555452 ; 22072964$ \\
\hline ncRv10932Ac & 1041165 & 1041129 & - & 4 & $20181675 ; 22072964$ \\
\hline ncRv11051c (MTS0823) & 1175225 & 1175315 & + & 2 & $22072964 ; 20181675$ \\
\hline ncRv11075A & 1200555 & 1200605 & + & 1 & 22072964 \\
\hline ncRv11147Ac (MTS0903) & 1275549 & 1276297 & - & 8 & 20181675 \\
\hline ncRv11160A & 1287126 & 1287201 & + & 13 & 23284830 \\
\hline ncRv11174Ac & 1306073 & 1306038 & - & 3 & 20181675 \\
\hline ncRv1222A & 1365274 & 1365365 & + & 5 & 22452820 \\
\hline ncRv11248c & 1393055 & 1393140 & + & 2 & $20181675 ; 22072964$ \\
\hline ncRv11296A & 1453007 & 1453060 & + & 13 & 20181675 \\
\hline ncRv11435c & 1612987 & 1613047 & + & 1 & 23284830 \\
\hline ncRv11534A & 1735693 & 1735747 & + & 15 & $19555452 ; 22072964$ \\
\hline ncRv1734A (MTS1338) & 1960667 & 1960783 & + & 7 & 24244498 \\
\hline ncRv11846Ac (Mrsl) & 2096839 & 2096768 & + & 4 & 29871950 \\
\hline ncRv2395A & 2692172 & 2692521 & + & 5 & 20181675 \\
\hline ncRv12560A & 2881252 & 2881320 & + & 2 & 20181675 \\
\hline ncRv12904A & 3214341 & 3214399 & + & 9 & 23284830 \\
\hline ncRv13003Ac & 3363153 & 3363023 & - & 2 & 20181675 \\
\hline ncRv13241Ac & 3621466 & 3621265 & - & 11 & 23284830 \\
\hline ncRv3418Ac & 3837458 & 3837288 & - & 13 & 23284830 \\
\hline ncRv13596A (MTS2774) & 4040879 & 4040938 & + & 5 & 20181675 \\
\hline ncRv13660Ac (MTS2822) & 4099478 & 4099386 & - & 13 & $20181675 ; 22072964$ \\
\hline ncRv13661A (MTS2823) & 4100669 & 4100968 & + & 15 & 20181675 \\
\hline
\end{tabular}

bacterial genomes using expression data $[18,19]$. However, the challenges while using such an approach include discriminating between sRNA expression signal and the noise arising from IGRs, and to systematically eliminate the signals which are associated with the neighbouring gene expression. We have devised a novel, moving-window based method for detecting sRNA expression in the IGRs. Our method is elegant in capturing both validated and novel sRNA expression, with reduced influence of the expression signals arising from both the upstream and the downstream gene UTRs. As RNA-Seq is used as the input, the same data allows for the simultaneous quantification of both protein-coding gene as well as sRNA expression. Using this method, we identified 119 IGR sRNAs in the mid-exponential growth phase, which also exhibit preferential binding for the transcription machinery.

Mycobacteria encounter diverse environments in the host such as nutrient depletion, hypoxia and iron limitation. Profiling of sRNAs in multiple conditions is therefore essential to understand the expression dynamics of
sRNAs, which correlates with the conditional responses of the cell. Our extended analysis of the repertoire of RNA-Seq data to detect sRNA expression revealed context-dependent expression of many sRNAs. As case studies, we chose some of these novel sRNAs identified by our method and attempted to explain their potential regulatory role by predicting gene targets. One such sRNA is ncRv11806 which shows expression in the stationary phase of growth. Resuscitation promoting factor (RpfB) is one of its predicted gene targets which is required for the revival of dormant bacteria. It is interesting to note that the binding of ncRv11806 to the $r p f B$ mRNA masks the $5^{\prime}$ UTR and the start codon, which likely hinders translation. Also, ncRv11706A appears as the hypoxia-induced persistence specific sRNA. One of the targets for ncRv11706A is $r e c X$, the expression of which is downregulated in the latent mycobacterial infection. By inspecting the putative binding sites, it appears that the sRNA ncRv11706A masks the start codon on the recX mRNA. Therefore, our methodology for identifying sRNAs and subsequent cataloguing of their 

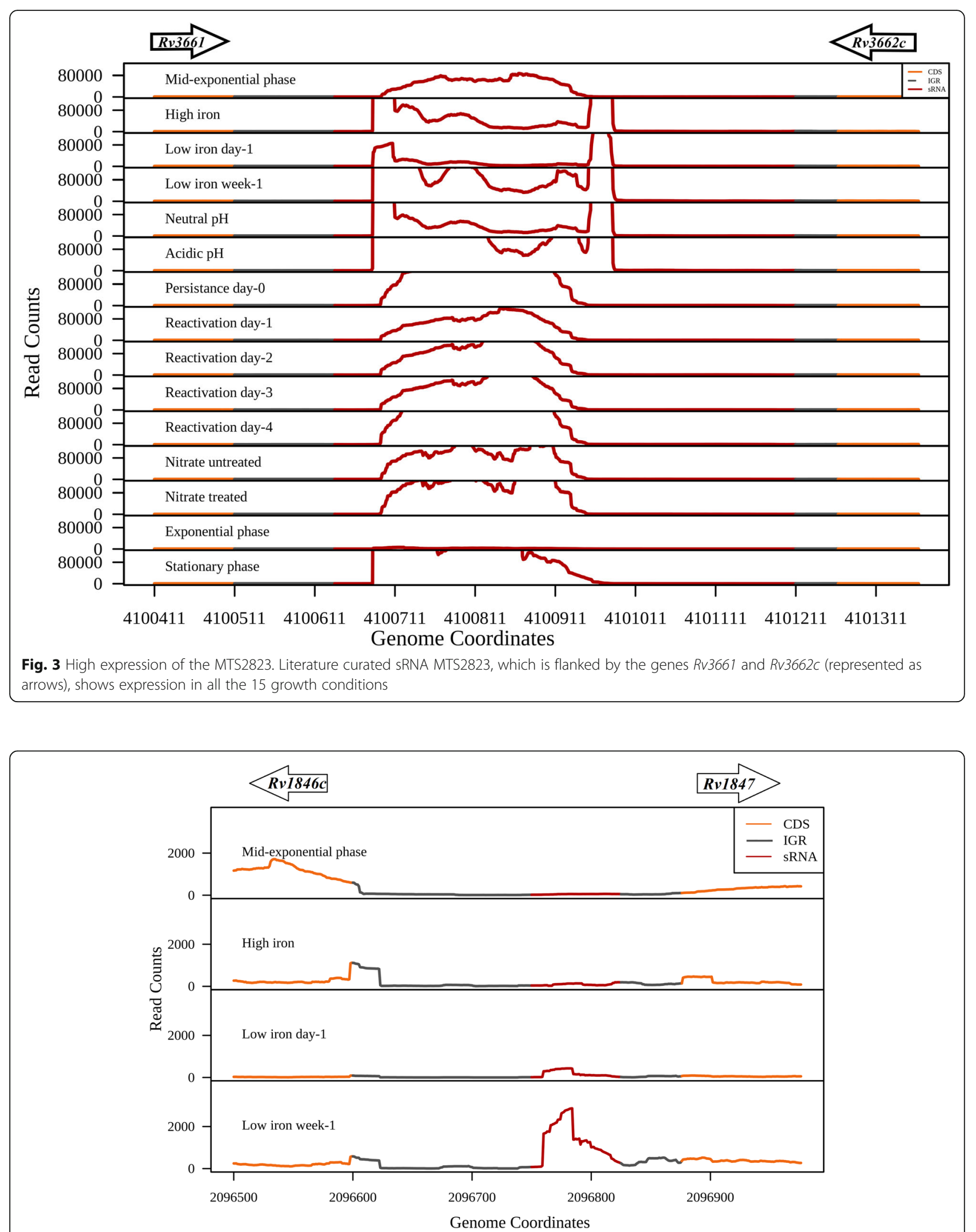

Fig. 4 Expression of Mrsl in iron rich and iron limiting conditions. sRNA Mrsl expression is induced in low iron conditions, both at day-1 and week-1,compared to iron-rich growth conditions. The upstream and the downstream genes are represented as arrows 


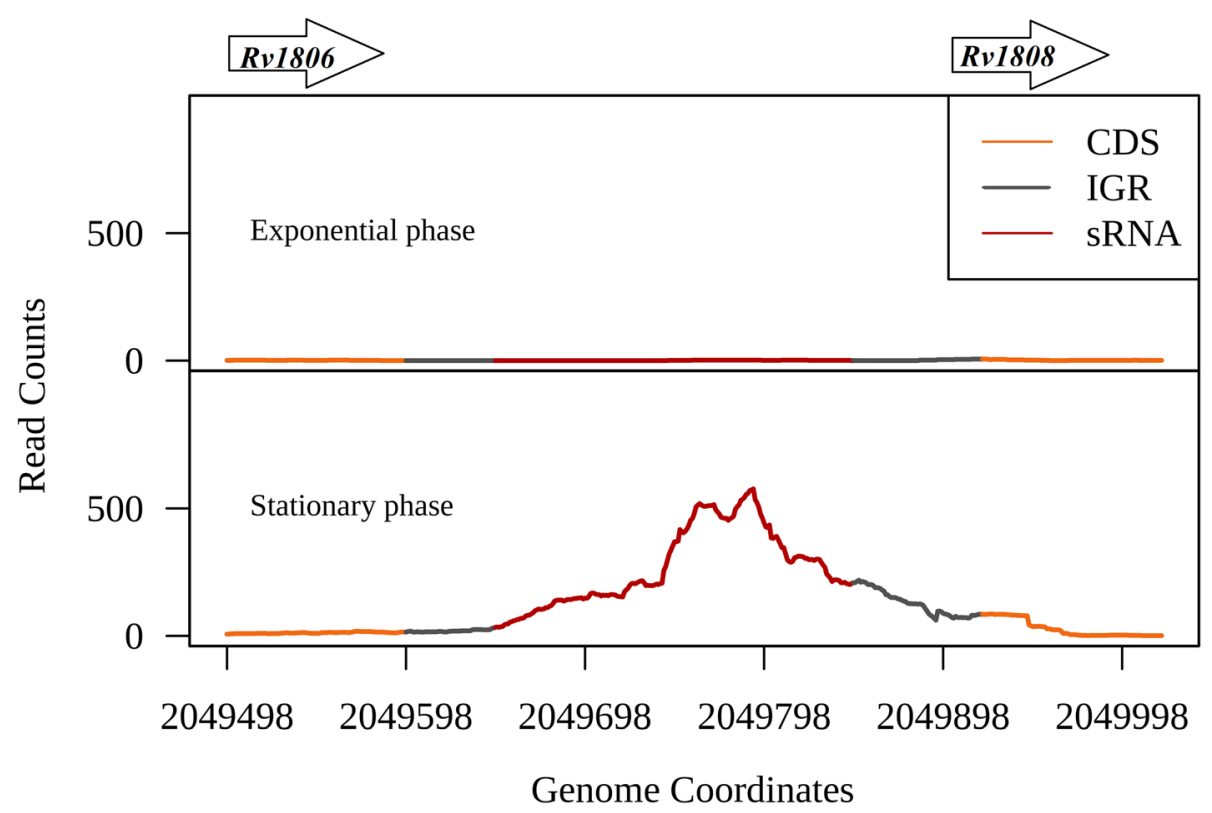

Fig. 5 Expression of ncRv11806 in the exponential and stationary phases of growth. sRNA ncRv11806 expression is induced in the stationary phase compared to the exponential growth phase. nCRv11806 lies in the IGR between the genes PE20 and PPE32 (represented as arrows)

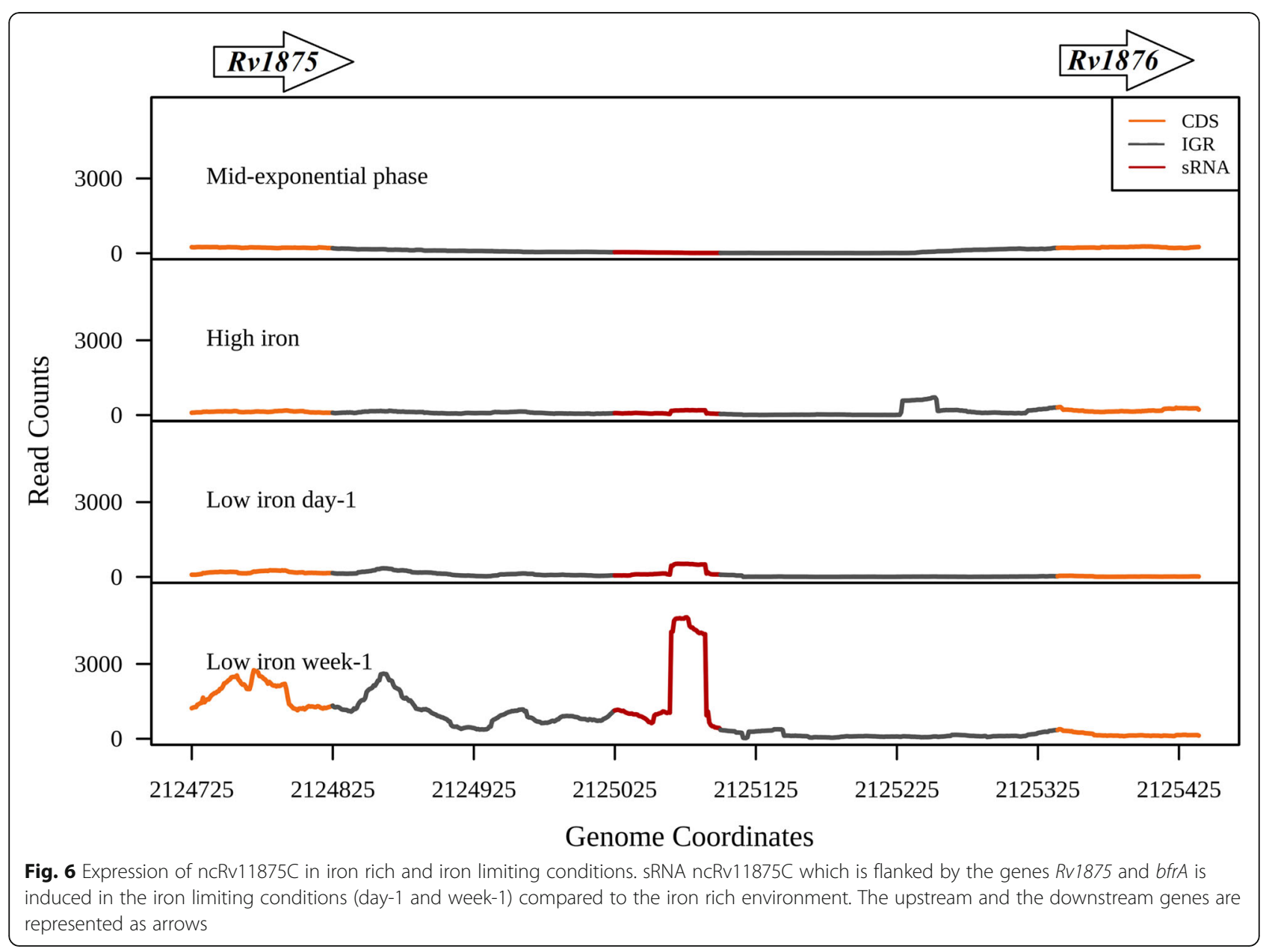




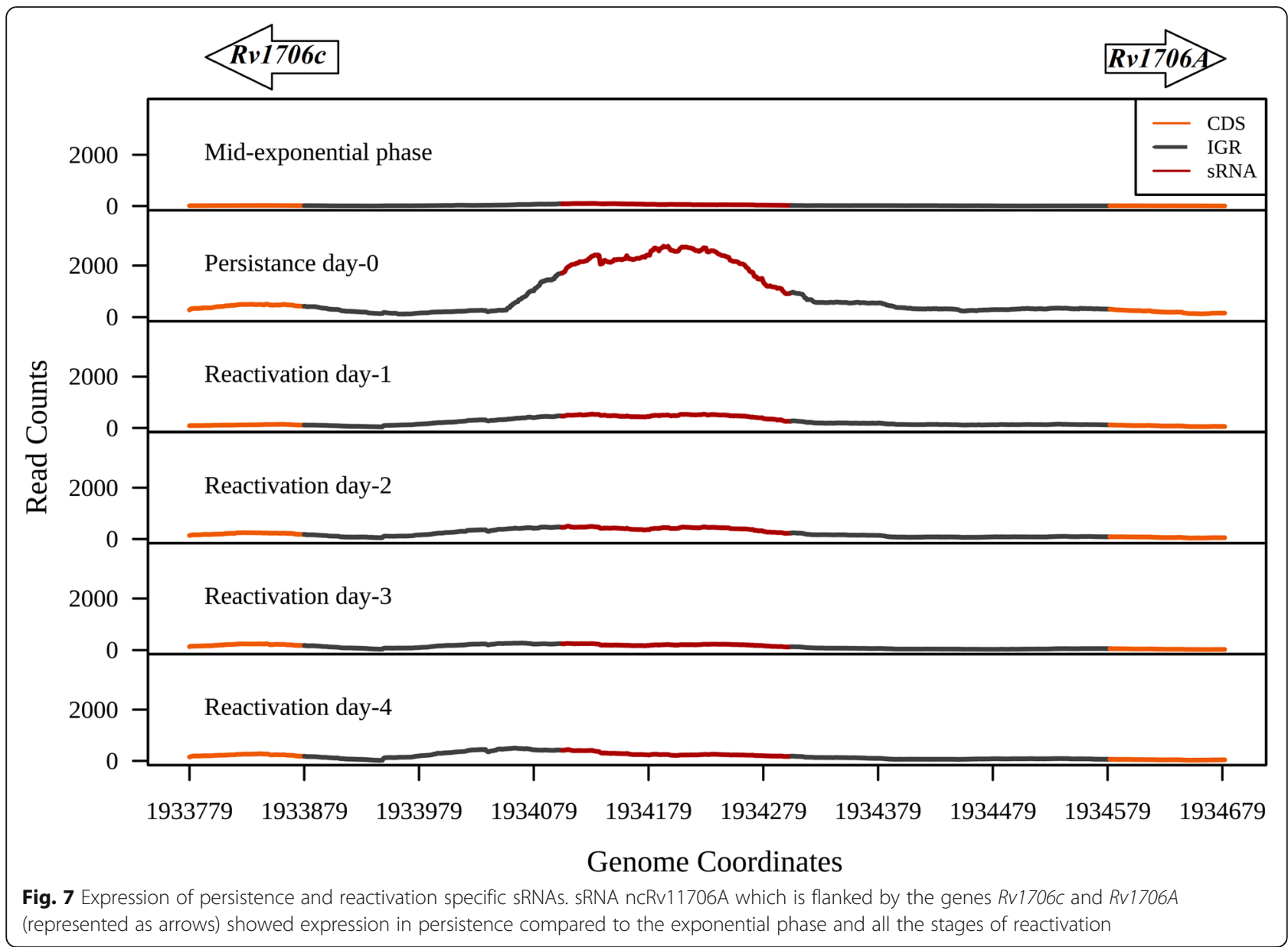

context-dependent expression generated novel perspectives on the sRNA mediated regulation. Further experimental validation of these putative sRNAs and their regulatory mechanisms will provide more insights on $M$. tuberculosis host adaptation and pathogenesis.

\section{Methods}

\section{Downloading and processing expression data}

RNA-Seq data of $M$. tuberculosis $\mathrm{H} 37 \mathrm{Rv}$ were retrieved from the NCBI-Sequence Read Archive (NCBI-SRA) (https://www.ncbi.nlm.nih.gov/sra) and European Nucleotide Archive (ENA) (https://www.ebi.ac.uk/ena) databases. Based on the library size quality, 5 different studies with 15 unique growth conditions were chosen for the analysis (Additional file 8: Table S1). SRA files were converted to fastq using fastq-dump available in sra-toolkit.2.1.18. The adapter sequences and the reads with the mean Phred score $<15$ were removed using trimmomatic (v 0.36) [36]. Trimmed data were aligned to the M. tuberculosis H37Rv reference genome (NC_000962.3) [37] using bowtie2 (v 2.1.0) [38] and the resulting SAM files were converted into
BAM files. BAM files for the replicate datasets were merged, sorted and indexed using samtools (v 0.1.18) [39].

\section{Selecting IGRs for the analysis}

Intergenic region (IGR) is defined as the genomic region between two protein-coding regions, and absolute intergenic region (AbIGR) is the IGR devoid of the UTRs. The genome size of M. tuberculosis H37Rv is 4411532 bp, which consists of 4018 proteins coding regions, 3 rRNAs and 45 tRNAs [37]. Of the 4017 Intergenic regions (IGRs), following were excluded from the analysis: i) 830 IGRs between operonic genes [40] ii) 48 IGRs encoding tRNAs and rRNAs [37] iii) 41 IGRs which contain mycobacterium specific repeat regions such as MIRU, VNTR [41] iv) 46 IGRs with Insertion elements such as IS6110 (16 copies), IS1018 (6 copies), REP13E12 (14 copies) and other IS (11 copies) [37] and v) IGRs which have a length smaller than $100 \mathrm{bp}$. This resulted in a total of 1037 IGRs for further analysis.

Genome coordinates of the protein-coding regions, tRNAs, rRNAs and repeat regions were retrieved from the 
NCBI Refseq gene annotation file (https://www.ncbi.nlm. nih.gov/refseq/) [37]. Transcription termination (3'UTR) coordinates were derived from the WebGeSTer database [42]. Coordinates of the transcription start site were obtained from the study by Cortes et al. [43]. From the intergenic regions, both the $3^{\prime}$ and the $5^{\prime}$ UTRs were removed to get the absolute intergenic region (AbIGR). MultiBamcov in Bedtools was used to obtain read counts which were further normalised using RPKM (Reads Per Kilobase Million) [44]. In the mid-exponential phase growth culture, top 1000 genes with high expression, which correspond to more than third quartile $(\mathrm{Q} 3)$ of the dataset were identified as highly expressed (HE) genes [21]. Similarly, bottom 1000 genes with the least expression, which correspond to less than first quartile $(\mathrm{Q} 1)$ of the dataset were identified as less expressed (LE) genes [21]. For comparison, experimentally validated sRNAs in $M$. tuberculosis were curated from the available literature (Additional file 8: Table S2).

\section{Identifying IGR expression}

To identify significant expression in the IGRs, windows of 50 base length with 25 bases sliding were used. In each IGR, a window was termed expressed if a) its expression value (RPKM) is greater than or equal to three times the median expression of the IGRs which are devoid of operons and repeat regions, and b) is greater than the adjacent windows. The first and the last windows in each IGR were masked to avoid the misclassification of the UTR expression as sRNA expression. The windows which overlap with the annotated pseudogenes were excluded. The expressing windows were merged to obtain putative sRNAs if they appear as contiguous in terms of genomic coordinates.

\section{ChIP-Seq data analysis}

Differential binding of RNAP and NusA in the expressed IGRs in mid-exponential phase was analysed using ChIP-Seq datasets GSM1003214 and GSM1003222, respectively [27]. These were compared with the ChIP-Seq data of the transcription factor Rv1186 (SRR1524124) and a genomic control (GSM2683113). Fastq-dump function of the sra-toolkit-2.1.18 was used to retrieve fastq format of the data. Trimmomatic (v 0.36) was used to trim the adapter sequences and the reads with the mean Phred score $<15$ [36]. The data were aligned to the reference genome using bowtie2 ( $\mathrm{v}$ 2.1.0) [38]. Aligned SAM files were converted to BAM files which were further sorted and indexed by samtools. From the sorted bam files, readcounts were obtained using multiBamCov of bedtools [44], which were further normalised to RPKM values to represent the binding profile. Welch Two Sample t-test was performed using R (https://www. r-project.org/) to compare the binding of transcription machinery to the expressed sRNAs encoding regions and the non-expressed IGRs.

\section{Gene targets of the identified sRNAs}

Genome-wide putative termination sites were derived using iTerm-PseKNC which were further used to determine the strands of the expressed sRNAs [45]. Potential gene targets of the sRNAs were derived using target prediction tools IntaRNA and TargetRNA2 [46, 47]. The reference genome of $M$. tuberculosis (NC_000962.3) was used as a target input file. Of the list of predicted targets with $p$-value $<0.05$, we considered 10 genes with minimum binding energy and the least p-value as potential targets for further analysis. Expression of the target genes in each of the 15 RNA-Seq data was quantified using bedtools [44]. The read counts were normalised by calculating RPKM.

All statistical analyses were performed using $\mathrm{R}$ (https://www.r-project.org/). The statistical significance of the conditional expression of sRNAs was assessed using Wilcoxon signed rank test. Data were analysed using in-house shell and python scripts. Circos version0.69 was used to represent the IGR expression along the circular chromosome co-ordinates [48].

\section{Supplementary information}

Supplementary information accompanies this paper at https://doi.org/10 1186/s12864-020-6573-5.

\begin{abstract}
Additional file 1 Figure S1. Expression profile of different functional categories in the mid-exponential growth phase. sRNAs are expressed on par with highly expressed and known essential genes. IGRs and AbIGRs, which are the potential sRNA encoding regions, also show significant expression suggesting functional relevance of the non-protein coding regions. (CDS - Protein coding regions, EG - Essential genes, HE - Highly expressed genes, LE - Less expressed genes, IGR - Intergenic regions, AbIGR - Absolute intergenic region devoid of neighbouring gene UTRs, and sRNA - curated sRNAs).
\end{abstract}

Additional file 2 Figure S2. Length distribution of 1037 IGRs chosen for the study. The IGR length ranges from $100 \mathrm{bp}$ to $1500 \mathrm{bp}$.

Additional file $\mathbf{3}$ Figure S3. Moving-window approach to identify sRNA expression. The IGR between the two protein-coding regions (CDS) including untranslated regions (5'UTR and $3^{\prime}$ UTR) was slided with windows of length 50 base with 25 bases sliding. In this cartoonic representation, the genomic region covered by the 3 rd window is predicted to encode an sRNA as it shows expression higher than the cut-off (the solid line) and the expression greater than the adjacent windows.

Additional file 4 Figure S4. Expression of IGRs across growth conditions. (A) Number of sRNAs expressed in each of the growth conditions studied (B) Frequency distribution of the number of sRNAs versus the number of the expressed growth conditions. Of the 430 sRNAs, 6 showed expression in all the 15 growth conditions and 48 sRNAs were expressed in more than 10 growth conditions.

Additional file $\mathbf{5}$ Figure S5. Expression of the predicted target genes of $n c R v 11806$.(A) Expression of the predicted gene targets of the sRNA ncRv11806 quantified in both exponential and stationary phases of growth. (B) Base pairing of ncRv11806 and one of its targets Rv1009. The $5^{\prime} U T R$ region of $r p f B$ is underlined and the start codon AUG is highlighted in bold letters. 
Additional file $\mathbf{6}$ Figure S6. Expression of the target genes of ncRv11875C. Expression of some of the predicted gene targets of the sRNA ncRv11875C which are differentially expressed in low iron conditions compared to mid-exponential and high iron growth.

Additional file $\mathbf{7}$ Figure S7. Expression of the target genes of ncRv11706A. (A) Expression of few of the target genes of ncRv11706A which are differentially expressed at persistence day-0 compared to midexponential growth phase and various time points of reactivation. (B) Base pairing of ncRv11706A and one of its targets recX. The start codon is highlighted in bold letters.

Additional file 8 Table S1. List of the RNA-Seq data. Description of the 15 RNA-Seq datasets used in the study along with their SRA study and accession numbers, growth conditions, and the data type. Table S2. Experimentally validated sRNAs. List of literature curated M. tuberculosis sRNAs which are experimentally validated.

Additional file $\mathbf{9}$ Table S3. Highly expressed and less expressed genes in the mid-exponential growth phase. List of highly expressed (a) and less expressed genes (b) protein coding genes in the mid-exponential growth phase with their RPKM values and the gene coordinates.

Additional file $\mathbf{1 0}$ Table S4. Context-dependent expression of the sRNAs. (a) List of 119 sRNAs expressed in the mid-exponential growth phase. (b) Context-dependent expression of all the 430 sRNAs derived by our method. (c) Binary matrix representation of the context-dependent expression of sRNAs across 15 growth conditions. (d) Expression matrix of the identified sRNAs in all the 15 growth conditions.

Additional file 11 Table S5. Target genes of some of the sRNAs. Predicted gene targets of the sRNAs along with their quantified expression in relevant growth conditions. Targets of (a) ncRv11806, (b) ncRv11875 and (c) ncRv11706A.

\section{Abbreviations}

AbIGR: Absolute Intergenic Region; CDS: Protein coding genes; ChIPSeq: Chromatin Immunoprecipitation sequencing; IGR: Intergenic regions; RNAP: RNA Polymerase; RPKM: Reads Per Kilobase Million; sRNA: Small RNAs; UTR: Untranslated Regions

\section{Acknowledgements}

Not applicable.

\section{Authors' contributions}

SRH conceived the study, VKGA, RB and SRH carried out the analysis, interpreted the data and wrote the manuscript. All authors read and approved the final manuscript.

\section{Funding}

This work was supported by INSPIRE Faculty Award by the Department of Science and Technology, Government of India to SRH and intra-mural funding of the Institute of Bioinformatics and Applied Biotechnology by the Department of IT, BT and S\&T of the Government of Karnataka.

\section{Availability of data and materials}

All the information relevant to the data analysed in this study are available in the Additional file 8 .

\section{Ethics approval and consent to participate}

Not applicable.

\section{Consent for publication}

Not applicable.

\section{Competing interests}

The authors declare that they have no competing interests.

Received: 23 October 2019 Accepted: 10 February 2020

Published online: 18 February 2020

\section{References}

1. World Health Organisation. Global Health TB Report. 2018.
2. Parrish NM, Dick JD, Bishai WR. Mechanisms of latency in Mycobacterium tuberculosis. Trends Microbiol. 1998;6:107-12.

3. Flentie K, Garner AL, Stallings CL. Mycobacterium tuberculosis transcription machinery: ready to respond to host attacks. J Bacteriol. 2016;198:1360-73.

4. Waters LS, Storz G. Regulatory RNAs in Bacteria. Cell. 2009;136:615-28. https://doi.org/10.1016/j.cell.2009.01.043.

5. Gripenland J, Netterling S, Loh E, Tiensuu T, Toledo-Arana A, Johansson J. RNAs: regulators of bacterial virulence. Nat Rev Microbiol 2010;8:857. https:// doi.org/10.1038/nrmicro2457.

6. Papenfort K, Vogel J. Regulatory RNA in bacterial pathogens. Cell Host and Microbe. 2010;8:116-27.

7. Masse E, Vanderpool CK, Gottesman S. Effect of RyhB small RNA on global Iron use in. Pharmacia. 2005;187:6962-71.

8. McCullen CA, Benhammou JN, Majdalani N, Gottesman S. Mechanism of positive regulation by DsrA and RprA small noncoding RNAs: pairing increases translation and protects rpoS mRNA from degradation. J Bacteriol. 2010;192:5559-71.

9. Sonnleitner E, Abdou L, Haas D. Small RNA as global regulator of carbon catabolite repression in Pseudomonas aeruginosa. Proc Natl Acad Sci. 2009; 106:21866-71.

10. Arnvig KB, Young DB. Identification of small RNAs in Mycobacterium tuberculosis. 2009:73 July:397-408.

11. Thomson NR, Houghton J, Boshoff HI, Arnvig KB, Croucher NJ, Rose G, et al. Sequence-Based Analysis Uncovers an Abundance of Non-Coding RNA in the Total Transcriptome of Mycobacterium tuberculosis 2011;7.

12. Moores A, Riesco AB, Schwenk S, Arnvig KB. Expression, maturation and turnover of DrrS, an unusually stable, DosR regulated small RNA in Mycobacterium tuberculosis. PLoS One. 2017;12:1-27.

13. Solans L, Gonzalo-Asensio J, Sala C, Benjak A, Uplekar S, Rougemont J, et al. The PhoP-dependent ncRNA Mcr7 modulates the TAT secretion system in Mycobacterium tuberculosis. PLoS Pathog. 2014;10.

14. Gerrick ER, Barbier T, Chase MR, Xu R, François J, Lin VH, et al. Small RNA profiling in Mycobacterium tuberculosis identifies Mrsl as necessary for an anticipatory iron sparing response. Proc Natl Acad Sci. 2018;115:6464-9.

15. Houghton J, Cortes T, Schubert O, Rose G, Rodgers A, de Ste CM, et al. A small RNA encoded in the Rv2660c locus of Mycobacterium tuberculosis is induced during starvation and infection. PLoS One. 2013;8:e80047.

16. Argaman L, Hershberg R, Vogel J, EGH W, Altuvia HM, Altuvia S. Novel small RNA-encoding genes in the intergenic regions of Escherichia coli. Prog Nuc Energy. 2001;38:327-30.

17. Tsai C-H, Liao R, Chou B, Palumbo M, Contreras LM. Genome-wide analyses in Bacteria show small-RNA enrichment for Long and conserved Intergenic regions. J Bacteriol. 2015;197:40-50.

18. Raghavan R, Groisman EA, Ochman H. Genome-wide detection of novel regulatory RNAs in E. coli. Genome Res. 2011;21:1487-97.

19. Wang M, Fleming J, Li Z, Li C, Zhang H, Xue Y, et al. An automated approach for global identification of sRNA-encoding regions in RNA-Seq data from Mycobacterium tuberculosis. Acta Biochim Biophys Sin. 2016;48: 544-53.

20. Liu Y, Orsi RH, Boor KJ, Wiedmann M, Guariglia-Oropeza V. An advanced bioinformatics approach for analyzing RNA-seq data reveals sigma $\mathrm{H}$ dependent regulation of competence genes in listeria monocytogenes. BMC Genomics 2016;17:1-11. doi:10.1186/s12864-016-2432-9.

21. Płociński P, Macios M, Houghton J, Niemiec E, Płocińska R, Brzostek A, et al. Proteomic and transcriptomic experiments reveal an essential role of RNA degradosome complexes in shaping the transcriptome of Mycobacterium tuberculosis. Nucleic Acids Res. 2019;47:5892-905.

22. DeJesus MA, Gerrick ER, Xu W, Park SW, Long JE, Boutte CC, et al. Comprehensive Essentiality Analysis of the Mycobacterium tuberculosis Genome via Saturating Transposon Mutagenesis . mBio. 2017:8:1-17.

23. Sassetti CM, Rubin EJ. Genetic requirements for mycobacterial survival during infection. Proc Natl Acad Sci. 2003;100:12989-94.

24. Dutta NK, Mehra S, Didier PJ, Roy CJ, Doyle LA, Alvarez X, et al. Genetic requirements for the survival of tubercle bacilli in primates. J Infect Dis. 2010;201:1743-52.

25. Rengarajan J, Bloom BR, Rubin EJ. From the cover: genome-wide requirements for Mycobacterium tuberculosis adaptation and survival in macrophages. Proc Natl Acad Sci. 2005;102:8327-32.

26. DiChiara JM, Contreras-Martinez LM, Livny J, Smith D, McDonough KA Belfort M. Multiple small RNAs identified in Mycobacterium bovis BCG are 
also expressed in Mycobacterium tuberculosis and Mycobacterium smegmatis. Nucleic Acids Res. 2010:38:4067-78.

27. Uplekar S, Rougemont J, Cole ST, Sala C. High-resolution transcriptome and genome-wide dynamics of RNA polymerase and NusA in Mycobacterium tuberculosis. Nucleic Acids Res. 2013;41:961-77.

28. Vogel U, Frank K. NusA Is Required for Ribosomal Antitermination and for Modulation of the Transcription Elongation Rate of both Antiterminated RNA and mRNA *. 1997;272:12265-71.

29. Greenblatt J, McLimont M, Hanly S. Termination of transcription by nusA gene protein of Escherichia coli. Nature. 1981;292:215-20. https://doi.org/10, 1038/292215a0.

30. Cunningham-Bussel A, Zhang T, Nathan CF. Nitrite produced by Mycobacterium tuberculosis in human macrophages in physiologic oxygen impacts bacterial ATP consumption and gene expression. Proc Natl Acad Sci U S A. 2013;1 10:E4256-65. https://doi.org/10.1073/pnas.1316894110.

31. Du P, Sohaskey CD, Shi L. Transcriptional and physiological changes during Mycobacterium tuberculosis reactivation from non-replicating persistence. Front Microbiol. 2016;7:1346. https://doi.org/10.3389/fmicb.2016.01346

32. Tufariello JAM, Jacobs WR, Chan J. Individual Mycobacterium tuberculosis resuscitation-promoting factor homologues are dispensable for growth in vitro and in vivo. Infect Immun. 2004;72:515-26.

33. Benjak A, Uplekar S, Zhang M, Piton J, Cole ST, Sala C. Genomic and transcriptomic analysis of the streptomycin-dependent Mycobacterium tuberculosis strain 18b. BMC Genomics. 2016;17:190. https://doi.org/10.1186/ s12864-016-2528-2.

34. Forse LN, Houghton J, Davis EO. Enhanced expression of recX in Mycobacterium tuberculosis owing to a promoter internal to recA. Tuberculosis. 2011;91:127-35. https://doi.org/10.1016/j.tube.2010.11.002.

35. Vogel J, Sharma CM. How to find small non-coding RNAs in bacteria. Biol Chem. 2005;386:1219-38.

36. Bolger AM, Lohse M, Usadel B. Trimmomatic: a flexible trimmer for Illumina sequence data. Bioinformatics. 2014;30:2114-20.

37. Cole ST, Brosch R, Parkhill J, Garnier T, Churcher C, Harris D, et al. Deciphering the biology of Mycobacterium tuberculosis from the complete genome sequence. Nature. 1998;393:537-44. https://doi.org/10.1038/31159.

38. Langmead B, Salzberg SL. Fast gapped-read alignment with bowtie 2. Nat Methods. 2012;9:357-9. https://doi.org/10.1038/nmeth.1923.

39. Subgroup 1000 Genome Project Data Processing, Wysoker A, Handsaker B, Marth G, Abecasis G, Li H, et al. The Sequence Alignment/Map format and SAMtools. Bioinformatics. 2009;25:2078-9. https://doi.org/10.1093/ bioinformatics/btp352.

40. Taboada B, Ciria R, Martinez-guerrero CE, Merino E. ProOpDB : Pro karyotic Op eron D ata B ase 2012;40 November 2011:627-31.

41. Supply P, Lesjean S, Gicquel B, Mazars E, Locht C, Vincent V. Variable human minisatellite-like regions in the Mycobacterium tuberculosis genome. Mol Microbiol. 2000;36:762-71.

42. Mitra A, Kesarwani AK, Pal D, Nagaraja V. WebGeSTer DB-A transcription terminator database. Nucleic Acids Res. 2011;39(SUPPL. 1):129-35.

43. Cortes T, Schubert OT, Rose G, Arnvig KB, Comas I, Aebersold R, et al. Genome-wide mapping of transcriptional start sites defines an extensive leaderless Transcriptome in Mycobacterium tuberculosis. Cell Rep. 2013;5: $1121-31$.

44. Quinlan AR, Hall IM. BEDTools: a flexible suite of utilities for comparing genomic features. Bioinformatics. 2010;26:841-2. https://doi.org/10.1093/ bioinformatics/bta033.

45. Feng $C-Q$, Zhang Z-Y, Zhu X-J, Lin Y, Chen W, Tang H, et al. iTerm-PseKNC: a sequence-based tool for predicting bacterial transcriptional terminators. Bioinformatics. 2018;35:1469-77. https://doi.org/10.1093/bioinformatics/ bty827.

46. Mann M, Wright PR, Backofen R. IntaRNA 2.0: Enhanced and customizable prediction of RNA-RNA interactions. Nucleic Acids Res. 2017:45:W435-9.

47. Kery MB, Feldman M, Livny J, Tjaden B. TargetRNA2: identifying targets of small regulatory RNAs in bacteria. Nucleic Acids Res. 2014;42:124-9.

48. Connors J, Krzywinski M, Schein J, Gascoyne R, Horsman D, Jones SJ, et al. Circos : an information aesthetic for comparative genomics. Genome Res. 2009;19:1639-45. https://doi.org/10.1101/gr.092759.109.19.

\section{Publisher's Note}

Springer Nature remains neutral with regard to jurisdictional claims in published maps and institutional affiliations.

\section{Ready to submit your research? Choose BMC and benefit from}

- fast, convenient online submission

- thorough peer review by experienced researchers in your field

- rapid publication on acceptance

- support for research data, including large and complex data types

- gold Open Access which fosters wider collaboration and increased citations

- maximum visibility for your research: over $100 \mathrm{M}$ website views per year

At BMC, research is always in progress.

Learn more biomedcentral.com/submissions 